\title{
Crossmodal repetition effects in the mu rhythm indicate tactile mirroring during action observation
}

Michel-Pierre Colla-b* ${ }^{*}$ Geoffrey Bird ${ }^{c-d}$, Caroline Catmure and Clare Press ${ }^{f}$

aSchool of Psychology, Université Laval, Quebec City, Canada, G1V 0A6

${ }^{\mathrm{b}}$ Center for interdisciplinary research in rehabilitation and social integration (CIRRIS), Quebec City, Canada, G1M 2S8

cMRC Social, Genetic, and Developmental Psychiatry Centre, Institute of Psychiatry, King's College London, UK, SE5 8AF ${ }^{d}$ Institute of Cognitive Neuroscience, UCL, London, UK, WC1N 3AR eDepartment of Psychology, University of Surrey, Guildford, UK, GU2 7XH fDepartment of Psychological Sciences, Birkbeck, University of London, UK, WC1E 7HX

\section{*Corresponding author:}

Michel-Pierre Coll

Centre interdisciplinaire de recherche en réadaptation et intégration sociale (CIRRIS)

525 Boulevard Wilfrid-Hamel bureau H-1312

Quebec City, Quebec, Canada, G1M 2S8

Tel: 418-529-9141 \#6127

Michel-pierre.coll.1@ulaval.ca 


\section{Abstract}

3 Similar cortical activations during the experience and observation of touch suggest

4 the presence of a tactile mirroring system. However, the specificity of observation-

5 related activity - i.e. whether observation excites the same representations as

6 experience of that specific tactile stimulation - is still to be established.

$7 \quad$ Furthermore, central mu rhythms are attenuated during the experience and

8 observation of touch, and also during action observation and execution, making it

9 unclear whether they index processing of predominantly tactile or motor features of

10 observed actions. The present study used an EEG cross-modal repetition paradigm

11 to assess the relative tactile and motor specificity of mu attenuation during action

12 observation. Two experiments were carried out during which participants executed

13 and observed actions in alternation, and the repetition of either tactile or motor

14 features of the actions were manipulated. The mu signal over central electrodes

15 varied as a function of tactile repetition, consistent with the claim of a tactile

16 mirroring system and its reflection in the mu signal. Of note was the fact that mu

17 attenuation was sensitive only to manipulation of tactile - not motor - properties of

18 actions, suggesting that caution should be employed when interpreting mu effects

19 during action observation as reflective of motor mirroring.

21 Keywords: adaptation, mirror neurons, mu rhythm, somatosensory 
EEG tactile mirroring 3

3 When we observe others being touched, somatosensory cortical areas are active

4 (e.g. Keysers et al., 2004; Blakemore, Bristow, Bird, Frith, \& Ward, 2005; Bufalari,

5 Aprile, Avenanti, Di Russo, \& Aglioti, 2007; Ebisch et al., 2008; Schaefer, Heinze, \&

6 Rotte, 2012; Martinez-Jauand et al., 2012). In line with these findings, the alpha (7-

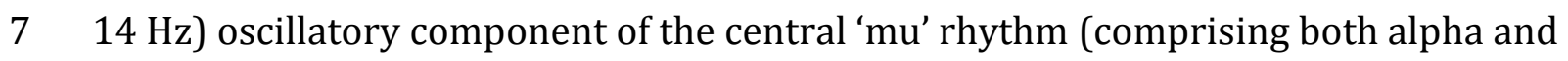

8 beta - 15-30 Hz - components; Hari \& Salmelin, 1997, although the term will be

9 used hereafter to refer solely to the alpha component) is attenuated both when

10 receiving (Cheyne et al., 2003; Gaetz \& Cheyne, 2006) and observing tactile

11 stimulation (Cheyne et al., 2003; Perry, Bentin, Bartal, Lamm, \& Decety, 2010; Höfle,

12 Pomper, Hauck, Engel, \& Senkowski, 2013). These findings suggest the presence of a

13 tactile mirroring system, whereby observation of touch activates representations

14 involved in processing the direct receipt of touch (note that our use of the word

15 'system' simply refers to similar distributed cortical representations activated in

16 observation and receipt conditions; Keysers, Kaas, \& Gazzola, 2010; Banissy \& Ward,

17 2007).

18

19 Attenuation of the central mu rhythm is also associated with both action

20 observation and execution (e.g. Cochin, Barthelemy, Roux, \& Martineau, 1999;

21 Muthukumaraswamy \& Johnson, 2004a, 2004b; Muthukumaraswamy, Johnson, \&

22 McNair, 2004). It is distinguishable from the classical occipital alpha signal by its

23 more anterior scalp distribution and the events which modulate it - namely, motor 
1 and tactile events as well as certain visual events (like action and touch

2 observation), rather than only visual events. This central mu attenuation when

3 observing and executing action has been assumed by many to reflect activity of the

4 human motor mirror system which processes observed actions in terms of

5 corresponding motor programmes required for execution (Cochin et al., 1999;

6 Muthukumaraswamy \& Johnson, 2004a, 2004b; Muthukumaraswamy et al., 2004;

7 Pineda, 2005; Oberman et al., 2005; Oberman, Pineda, \& Ramachandran, 2006;

8 Oberman, Ramachandran, \& Pineda, 2008; Lepage \& Théoret, 2006; Ulloa \& Pineda,

9 2007; Cheng et al., 2008; Rizzolati, Fabbri-Destro, \& Cattaneo, 2009; Ferrari et al.,

10 2012). However, if we indeed mirror observed touch, and mu oscillations can reflect

11 tactile processing, this assumption may be invalid. Specifically, central mu

12 oscillatory responses during action observation may instead reflect mirroring of the

13 tactile components of an action (e.g. what it feels like to grasp an object), rather than

14 the motor activity necessary to execute the action. This possibility is especially

15 plausible given that source localization of mu effects (Cheyne et al., 2003; Hari et al.,

16 1998; Rossi et al., 2002; although see van Wijk, Willemse, \& Vandertop, 2012), and

17 correlations between mu and BOLD responses (Ritter, Moosmann, \& Villringer,

18 2009; Arnstein, Cui, Keysers, Maurits, \& Gazzola, 2011), indicate that mu effects may

19 be generated in the somatosensory cortex.

21 However, to provide evidence that these oscillatory responses reflect mirroring

22 (either tactile or motor), specificity must be demonstrated. In the case of tactile

23 mirroring, observation of tactile stimulation must excite the same representations 
1 as experience of that specific tactile stimulation, rather than increase

2 somatosensory activity in a general, non-specific manner (see Oosterhof, Tipper, \&

3 Downing, 2013; Cook, Bird, Catmur, Press, \& Heyes, 2014). To demonstrate motor

4 mirroring, observation of action must excite the same motor representations

5 involved in performing that specific action.

6

7 Recently, cross-modal repetition fMRI designs have been used to support claims of

8 motor mirroring processes. Repetition suppression is the term used to describe the

9 reduction observed in the neural response when events activating the same

10 representation occur in succession. Cross-modal repetition suppression occurs

11 when observation of Action A causes a smaller response when preceded by

12 execution of Action A, than when preceded by execution of Action B. This pattern of

13 results is taken to indicate that both the observation and execution of Action A share

14 overlapping neural representation and therefore provides evidence of action

15 mirroring (Chong, Cunnington, Williams, Kanwisher, \& Mattingley, 2008; Grill-

16 Spector, Henson \& Martin, 2006; Kilner, Neal, Weiskopf, Friston, \& Frith, 2009;

17 Lingnau, Gesierich, \& Caramazza, 2009; Press, Weiskopf, \& Kilner, 2012). To our

18 knowledge, no studies have previously demonstrated cross-modal repetition effects

19 on mu oscillatory responses. However, of relevance to mirror system investigations,

20 Perry and Bentin (2009) used a unimodal EEG repetition design to show that

21 repeated observation of the same grasp type resulted in reduced mu attenuation

22 when compared to observation of different grasp types (see also Ortigue,

23 Thompson, Parasuraman \& Grafton, 2009). 
2 At present, evidence for specific tactile mirroring beyond somatotopic matching (e.g.

3 Blakemore et al., 2005; Kuehn, Muller, Turner, \& Schütz-Bosbach, 2014) has not

4 been obtained with any neuroimaging measure. Additionally, as noted above,

5 specificity has not been investigated in mu attenuation, meaning that conclusions

6 concerning its capability to index mirror processes - either tactile or motor - are

7 premature. The specificity of responses during the observation of tactile stimulation,

8 as well as relative contributions of tactile and motor mirroring to mu attenuation

9 during action observation, was assessed in two experiments using an EEG cross-

10 modal repetition design. Power in the alpha frequency range was measured over

11 sensorimotor areas in response to repeated or non-repeated actions where

12 repetition was defined according either to the tactile properties of an object

13 (Experiment 1) or the motor, tactile, or both motor and tactile, features of the action

14 (Experiment 2).

15

\section{2. Materials and methods}

\section{$18 \quad 2.1$ Participants}

20 Seventeen healthy participants took part in Experiment 1 ( 9 females, mean age $=$

21 25.82, range 19-43). Fifteen new participants ( 3 females, mean age $=27.70$ years,

22 range 18-41) took part in Experiment 2. Two participants in Experiment 1, and

23 three participants in Experiment 2 were replacements for participants where more 
1 than $40 \%$ of trials were excluded (see below). All participants gave informed

2 consent to participate in exchange for course credit or monetary compensation, had

3 normal or corrected to normal vision and were right-handed as assessed by the

4 Edinburgh Handedness Inventory (Oldfield, 1971). Both studies were approved by

5 the Research Ethics Committee within the Department of Psychological Sciences,

6 Birkbeck, University of London.

7

\section{$8 \quad 2.2$ Aims and study design}

10 Experiments 1 and 2 used a crossmodal repetition design to test for tactile and

11 motor specificity in mu attenuation during action observation. Specifically, mini-

12 blocks were presented in which action observation and execution alternated. In

13 Repeat mini-blocks, actions with the same tactile or motor properties were

14 observed and executed, while in Non-Repeat mini-blocks, different tactile or motor

15 properties were present in observation and execution. According to repetition logic

16 (e.g. Grill-Spector et al., 2006), if mu attenuation shows selectivity for specific tactile

17 sensation or actions, it should differ in Repeat and Non-Repeat blocks. Mini-blocks,

18 rather than single actions, were used as the unit of repetition because single cell

19 data suggest that repetition effects in motor mirror representations may be

20 cumulative (Kilner, Kraskov, \& Lemon, 2014).

22 Experiment 1 was designed to manipulate the tactile components of observed and

23 executed actions while holding the motor component constant across conditions. 
1 This was done by asking participants to carry out the same action (squeezing a ball)

2 on balls with different textures. Two identical rubber balls were used, but one had a

3 smooth texture and the other was covered in a black plastic mesh to alter its tactile

4 properties. Oscillatory activity in the alpha frequency range at central electrodes

5 was therefore examined in response to observation or execution of an action on a

6 ball of a certain texture (e.g. Smooth) as a function of whether it had been preceded

7 by observation or execution of an action on a ball with the same (Smooth) or a

8 different (Mesh) texture (see Table 1). Therefore, by contrasting Repeat vs. Non-

9 Repeat mini-blocks, we examined whether mu power in response to observing or

10 executing an action with certain tactile properties (e.g. squeeze a smooth ball)

11 varied as a function of whether it had been preceded by observation or execution of

12 an action with the same tactile properties (squeeze a smooth ball) or different

13 tactile properties (squeeze a mesh ball). In all mini-blocks, participants alternated

14 between observing and executing actions, and within a mini-block, observed and

15 executed tactile properties were held constant to equate within-modality repetition

16 (i.e. if in a mini-block participants executed actions on a Smooth ball and observed

17 actions on a Mesh ball, they would do so for the whole mini-block). If repetition

18 effects (change in oscillatory attenuation when a texture is repeated vs. not

19 repeated) are observed in this experiment, they must reflect mirroring of the tactile

20 rather than motor properties of the observed stimulus, because the motor

21 properties of the two observed squeezes are the same. To investigate whether an

22 action needs to be observed on the object in order to mirror its tactile properties,

23 the Tactile-Action condition was contrasted with a Tactile-Arrows condition in 
1 which attention was drawn towards the object, including its tactile properties, by

2 moving arrows rather than by a hand grasping the object.

4 Experiment 2 was designed to extend Experiment 1 by assessing the relative

5 contribution of tactile and motor mirroring to the mu attenuation during action

6 observation. This experiment used two action types: the same squeeze on a smooth

7 ball as in Experiment 1 (Squeeze) and making writing movements with a pen

8 (Write). In order to assess whether any observed specificity for an action reflects

9 mirroring of the motor or tactile components, three conditions presented variants

10 on the design outlined above. In the Motor+Tactile condition, participants observed

11 and executed Squeeze and Write actions directed towards objects in which both the

12 motor and the tactile components of the action were present. Squeeze actions

13 consisted of all fingers moving inwards and outwards to squeeze the ball (motor

14 component) and touch to proximal parts of all fingertips and the palm of the hand

15 (tactile component). Write actions consisted of fingers making repetitive downward

16 strokes (motor component) and touch to more distal aspects of the fingertips

17 (tactile component). Therefore, any repetition effects (change in oscillatory

18 attenuation when an action is repeated vs. not repeated) should be present

19 regardless of whether mu attenuation reflects mirroring of motor or tactile 20 properties.

22 In the Motor-Only condition, participants observed and executed the actions

23 without the presence of the objects. This condition therefore included the motor 
1 components of the actions, but there was an absence of cutaneous stimulation.

2 Finally, we included a condition where any repetition effects would reflect mirroring

3 of the tactile properties of the actions. In this Tactile-Only condition, participants

4 executed actions towards objects but observed videos where the hand did not move;

5 instead, the ball or pen was seen moving into the hand and making contact at the

6 same location as in the Motor+Tactile condition. Therefore, if oscillatory attenuation

7 reflects mirroring of motor properties, repetition effects will be seen in the

8 Motor+Tactile and Motor-Only conditions but not the Tactile-Only condition. If

9 oscillatory attenuation reflects mirroring of cutaneous touch, repetition effects will

10 be seen in the Motor+Tactile and Tactile-Only conditions but not the Motor-Only

11 condition.

\section{$13 \quad 2.3$ Visual stimuli}

15 All video clips were presented with E-Prime 2.0 Professional software (Psychology

16 Software Tools Inc., Sharpsburg PA, USA) on a 19 inch monitor ( 716 x 537 pixels)

17 located approximately $40 \mathrm{~cm}$ from the participant.

19 The Tactile-Action visual stimuli in Experiment 1 consisted of video clips of 1500 ms

20 duration during which a right hand squeezed either a yellow smooth ball or the

21 same ball covered in a black plastic mesh. In the Tactile-Arrows condition two

22 arrows were seen moving towards the same balls. The speed, location and distance

23 traversed by the arrows in the Tactile-Arrows condition were matched 
1 approximately to the kinematics of the index finger and thumb movements in the

2 Tactile-Action condition (each Tactile-Arrows video was matched to one of the

3 Tactile-Action videos). Four different actors (two females) performed the two

4 actions, for a total of 16 different stimuli.

5

6 The visual stimuli in Experiment 2 consisted of video clips as described above, of

71500 ms duration, during which a right hand executed actions on a black

8 background. Tactile-Only clips were developed using a string to move the objects

9 into the actor's hand. The string was subsequently edited out of each video clip

10 using Adobe Photoshop 5.0 software (Adobe Systems Inc, San Jose, CA, USA). Four

11 different actors (two females) generated the six video clips, resulting in a total of 24

12 different stimuli. Stills from the different video clips for both experiments are shown

13 in Figure 1.

\section{$15 \quad 2.4$ Procedure}

17 Participants sat comfortably in an electrically shielded, soundproofed and dimly lit

18 room. They were instructed to avoid any movements other than those prescribed in

19 the study, to keep eye blinks to a minimum and try to blink only in the inter-

20 stimulus intervals. During both experiments, the participant's right hand was

21 occluded from view with a box and movements were recorded with a webcam

22 placed at the end of the box. The video recording of the participant's hand was live 
1 monitored by an experimenter and mini-blocks with execution mistakes were

2 marked and later removed from the analyses.

4 At the beginning of Experiment 1, participants were asked to manipulate the two

5 balls (Smooth and Mesh) and describe each of them with five different words in

6 order to familiarize themselves with the different textures and appearances. The

7 two balls were then fixed within the occlusion box to allow for relatively error-free

8 action execution. Participants were instructed to execute the actions in response to

9 the presentation of a triangular cue presented on a black background for $1500 \mathrm{~ms}$.

10 Participants were to move their hand away from their body to perform a squeeze on

11 one ball (signalled by an upward pointing triangle) and towards their body to

12 perform the squeeze on the other ball (downward pointing triangle). The object

13 locations in the box were counterbalanced across participants. Participants were

14 asked to initiate the correct action only when they saw the triangle and to return to

15 the resting position by the time that the triangle disappeared. Reaction times were

16 not measured, but participants were asked to pace their action with the execution

17 cues. They were also warned after practice and between each session if monitoring

18 of their movements revealed that they executed actions excessively fast or slow.

19 There were four types of mini-block in Experiment 1 according to the two main

20 experimental factors: Repetition of texture (Repeat, Non-Repeat) and Condition

21 (Tactile-Action, Tactile-Arrows), and 12 mini-blocks of each type. 
1 The procedure in Experiment 2 was identical to that in Experiment 1 except for the

2 following: at the beginning of the mini-block, participants received the instruction to

3 execute the actions with or without objects. The same objects as those used in the

4 video clips (ball and pen) were fixed within the occlusion box and participants were

5 to execute the appropriate action in response to the triangle cue. In the

6 Motor+Tactile and Tactile-Only conditions, contact with the appropriate object was

7 made in these locations. In the Motor-Only condition, the participant's hand was

8 positioned away from the objects such that no contact was made. There were six

9 types of mini-block according to the two main experimental factors: Repetition

10 (Repeat, Non-Repeat) and Condition (Motor+Tactile, Motor-Only and Tactile-Only),

11 and 12 mini-blocks of each type.

13 At the beginning of each mini-block, participants first saw the instruction "Block

14 begin" (Experiment 1) for $5000 \mathrm{~ms}$ or the instruction indicating that they should

15 execute the actions with or without the objects in the following mini-block

16 (Experiment 2). These instructions were followed by 11 experimental trials (one

17 mini-block). For each experimental trial, the participants first saw a fixation cross

18 with a mean duration of $750 \mathrm{~ms}$ ( 500 to $1000 \mathrm{~ms}$ ) followed by the presentation of a

191500 ms video clip or execution cue, and then a black screen for an ISI of a mean

20 duration of $1750 \mathrm{~ms}$ (750 to $2750 \mathrm{~ms}$ ). The first action presented/executed and the

21 first trial type (observation or execution) were equally distributed in the mini-

22 blocks and the hand models were pseudo-randomized so that all models were

23 presented at least once in each mini-block. There was a $10 \mathrm{~s}$ interval between each 
1 mini-block, and the experimental procedure was divided into two (Experiment 1) or

2 three (Experiment 2) 25 minute sessions. The mini-block presentation order was

3 fully randomized within each session.

5 3. EEG recordings and analyses

\section{$7 \quad 3.1$ Recording and preprocessing}

9 EEG data was recorded at $500 \mathrm{~Hz}$ using Brain Vision Recorder (Brain Products

$10 \mathrm{GmbH}$, Munich, Germany) from 28 active $\mathrm{Ag} / \mathrm{AgCl}$ electrodes (Fp1, Fp2, F3, F4, F7,

11 F8, Fz, FC1, FC2, FC5, FC6, FCz, Cz, C3, C4, CP1, CP2, CP5, CP6, P3, P4, P7, P8, Pz, T7,

12 T8, 01, 02) mounted on an elastic cap (EasyCap, Brain Products GmbH, Munich,

13 Germany) according to the 10/20 international placement system (Jasper, 1958).

14 Three additional EOG channels were used to monitor vertical and horizontal eye

15 movements. Two were placed at one centimeter from the outer canthi of the left and

16 right eyes and the other one below the left eye. The signal was online filtered

17 between 0.1 and $80 \mathrm{~Hz}$ with acquisition reference at the left and right mastoids.

18 Electrode impedances were maintained at $10 \mathrm{k} \Omega$ or below.

20 All offline EEG analyses were performed with SPM8 (Wellcome Trust Centre for

21 Neuroimaging, London, UK, ww.fil.ion.ucl.ac.uk/spm). The data were first bandpass

22 filtered at 1 and $45 \mathrm{~Hz}$ and epoched at $-200 \mathrm{~ms}$ to $1700 \mathrm{~ms}$ relative to the beginning

23 of the video clip or execution cue of trials 2-11 in each mini-block (trial 1 was 
1 discarded given that we were interested in investigating repetition effects). The

2 same epoch duration was also extracted during a blank screen at the beginning of

3 each mini-block to serve as a baseline period during which no actions were executed

4 or observed. Trials were rejected if either the peak to peak amplitude of the trial

5 exceeded $135 \mu \mathrm{v}$ at any of the EEG or EOG channels (Experiment 1: 16\%,

6 Experiment 2: 10\%) or an incorrect movement was performed by the participant

7 during the mini-block (Experiment 1: $<1 \%$, Experiment 2: 2\%). More specifically, in

8 Experiment 1, 11 execution mistakes were noted (error rate of $0.0013 \%$ ) leading to

9 the removal of five experimental blocks from analyses ( $0.006 \%$ of the data). In

10 Experiment 2, 38 execution mistakes were noted (error rate $0.0035 \%$ ) leading to

11 the removal of 23 experimental blocks from analyses (2.12 \% of the data). Error rate

12 separated by Repetition condition shows a similar rate in Repeat trials $(0.0012 \%)$

13 and Non-Repeat trials (0.0014\%) in Experiment 1 as well as in Experiment 2

$14 \quad(0.003 \%$ and $0.004 \%$, for Repeat and Non-Repeat trials respectively). Rejection rate

15 was similar in all types of epoch. After artifact detection, the data were

16 downsampled to $100 \mathrm{~Hz}$ prior to analysis.

\section{$18 \quad 3.2$ Time-frequency analyses}

20 Spectral changes in oscillatory activity were analyzed using a Morlet wavelet

21 decomposition transform with a width of seven cycles per wavelet, across a 5-45 Hz

22 frequency range and -200 to $1700 \mathrm{~ms}$ relative to event onset in experimental trials

23 (the beginning of the video clip or the execution cue), or -2200 to -300 ms relative to 
1 the start of the block (baseline). The decomposition was performed for each trial,

2 electrode and participant. Time-frequency maps were averaged within each

3 experimental condition (or baseline period) using the SPM8 robust averaging

4 procedure (c.f. Garrido et al., 2009), and $\log 10$ transformed to normalize. All

5 experimental conditions were subsequently baseline corrected by subtracting the

6 average inter mini-block epoch, which was averaged across all such epochs in the

7 experiment independent of condition.

\section{$9 \quad 3.3$ Selection of sites and bands of interest}

11 All analyses were carried out on the centrally located electrodes C3, C4, CP1 and

$12 \mathrm{CP} 2$. The location of these sites has been consistently associated with mu

13 attenuation during movement execution and observation in previous studies (e.g.

14 Muthukumaraswamy \& Johnson, 2004a, 2004b; Oberman et al., 2005; Proverbio,

15 2012). Furthermore, t-tests also confirmed that these locations showed significant

$16 \mathrm{mu}$ attenuation relative to baseline during execution trials in the present

17 experiments (Experiment 1: $\mathrm{t}(14)=2.82, p=0.01, \mathrm{~d}=0.73$; Experiment 2: $\mathrm{t}(16)=$

$183.49, p=0.003, d=0.85$ ). To investigate potential interactions between the effects of

19 interest and laterality (although none were found), attenuation relative to baseline

20 was averaged separately at the two left $(\mathrm{C} 3, \mathrm{CP} 1)$ and the two right electrodes (C4,

21 CP2) in order to create a two level Hemisphere factor (Left/Right) that was included

22 in all statistical analyses. 
1 To take into account inter-individual differences in the range of the mu attenuation,

$23 \mathrm{~Hz}$ bands were chosen for each participant in the 7-14 $\mathrm{Hz}$ band. The selection was

3 made by averaging all execution trials 0 to $1500 \mathrm{~ms}$ post stimulus (for consistency

4 with main analysis, see below) in each experiment in all $3 \mathrm{~Hz}$ bands and choosing

5 the band where the attenuation was strongest at electrodes C3, C4, CP1 and CP2

6 (see Klimesch, 1999; Muthukumaraswamy \& Johnson, 2004a, 2004b; Babiloni et al.,

7 2009). This procedure led to an average mu band of 9-12 Hz for Experiment 1 and 8-

$811 \mathrm{~Hz}$ for Experiment 2 (range 7-10 to $11-14 \mathrm{~Hz}$ in both experiments ${ }^{1}$ ). This

9 selection criterion was orthogonal to any analyses of interest, which investigated

10 differences in such attenuation as a function of different factors.

\section{$12 \quad 3.4$ Effects of action type and repetition}

14 Two three-way repeated measures ANOVAs were then performed on the mu

15 attenuation relative to baseline in each experiment, for the stimulus period ( 0 to

161500 ms post stimulus). Experiment 1 ANOVAs included factors of Hemisphere

17 (Left, Right), Repetition (Repeat, Non-Repeat) and Condition (Tactile-Action and

18 Tactile-Arrows). Experiment 2 included the same factors, but Condition now had

19 three levels (Motor+Tactile, Motor-Only, Tactile-Only) and Repetition referred to

20 repetition of the action type rather than texture. The significance threshold was set

21 at $p<.05$ and a Greenhouse-Geisser correction was used when the sphericity

22 assumption was not met. 


\section{4. Results}

3 Results from all statistical analyses are presented in Table 2. Only results relevant to

4 the crossmodal repetition effects that this study aimed to measure are presented in

5 the following section.

\section{$7 \quad 4.1$ Experiment 1}

9 There was a significant main effect of Condition $\left[F(1,16)=9.03, p=0.01\right.$, partial $\eta^{2}=$

$10.36]$, indicating significantly stronger mu attenuation in the Tactile-Action condition

11 relative to the Tactile-Arrows condition. Of critical interest, there was also a

12 significant main effect of Repetition of texture $\left[F(1,16)=5.47, p=0.03\right.$, partial $\eta^{2}=$

13.26 ; see Fig. 2]. This effect is one of repetition suppression, whereby there was less

14 mu attenuation when the tactile properties had been repeated between observation

15 and execution. This repetition effect therefore indicates mirroring of tactile

16 properties reflected in the mu response. Attenuation relative to baseline as a

17 function of Repetition and Condition and Time frequency plots (7-14 Hz) for

18 Experiment 1 are shown in Figure 2.

\section{$21 \quad 4.2$ Experiment 2}

23 A marginally significant effect of Hemisphere $\left[F(1,14)=3.66, p=.08\right.$, partial $\eta^{2}=$ 
$\left.\begin{array}{ll}1 & .21\end{array}\right]$ indicated stronger mu attenuation on the left than the right. This trend is

2 potentially similar in nature to a marginally significant Hemisphere $\mathrm{x}$ Condition

3 interaction in Experiment $1\left[F(1,16)=2.60, p=0.13\right.$, partial $\left.\eta^{2}=.14\right]$, which

4 reflected stronger attenuation on the left for the Texture-Action condition only.

$5 \quad$ These marginal effects may reflect stronger left attenuation when observing and

6 executing actions with the right hand, but given that the effects (1) were only

7 marginal and (2) did not interact with repetition effects, they will not be discussed

8 further.

10 The main effect of Repetition was not significant $\left[F(1,14)=3.18, p=.10\right.$, partial $\eta^{2}=$

11 .19]. However, there was a significant Condition $\mathrm{x}$ Repetition interaction $[F(2,28)=$

$123.5, p=0.04$, partial $\eta^{2}=.20$ see Fig. 3]. This interaction was decomposed with three

13 planned two-tailed t-tests, assessing repetition effects in each of the conditions.

14 These tests revealed significantly greater mu attenuation during Repeat than Non-

15 Repeat mini-blocks in the Motor+Tactile $[t(14)=-2.28, p=0.04, d=.20]$ and Tactile-

16 Only $[t(14)=-2.94, p=0.01, d=.22]$ conditions, but not in the Motor-Only condition

$17[t(15)=0.61, p=0.55, d=.09]$. Therefore, there was a repetition effect in conditions

18 where the tactile component was repeated (Motor+Tactile and Tactile-Only) but not

19 the condition where only the motor component was repeated (Motor-Only).

20 Interestingly, in this experiment the effect is one of repetition enhancement rather

21 than suppression. Attenuation relative to baseline as a function of Repetition and

22 Condition and time frequency plots $(7-14 \mathrm{~Hz})$ for Experiment 2 are shown in Figure

233. 


\section{5. Discussion}

4 The present study first aimed to assess critically the existence of a tactile mirroring

5 system; whether the perception of tactile stimulation in others activates specific

6 representation(s) activated by the direct receipt of touch. To this end, an EEG cross-

7 modal repetition design was used (Kilner et al., 2009; Press, Catmur et al., 2012;

8 Press, Weiskopf, et al., 2012; Segaert, Weber, de Lange, Petersson, \& Hagoort, 2013)

9 and mu attenuation was measured at central electrodes. If the product of a tactile

10 mirroring system, mu attenuation should show specificity - activation of the same

11 tactile representation in the observer as that which is observed - and therefore

12 cross-modal repetition effects should be demonstrated when observation of tactile

13 stimulation is alternated with the same tactile stimulation in the participant.

14 Experiment 1 revealed crossmodal repetition effects in the mu signal in a design

15 where the motor components of observed and executed actions were held constant

16 but the tactile components were either repeated or not.

18 These results provide evidence for the presence of a tactile mirroring system and

19 add to other studies showing that similar patterns of somatosensory activation are

20 observed when an individual is touched and when that individual observes another

21 being touched (e.g. Keysers et al., 2004; Blakemore et al., 2005; Kuehn et al., 2014).

22 They are consistent with findings of mu attenuation when receiving and observing

23 touch (e.g. Cheyne et al., 2003; Gaetz \& Cheyne, 2006; Perry et al., 2010; Höfle et al., 
1 2013), and with evidence from studies of action execution suggesting that the mu

2 signal may largely originate from primary somatosensory cortex (Hari et al., 1998;

3 Cheyne et al., 2003; Rossi et al., 2002; Hari, 2006; Ritter et al., 2009; Arnstein et al.,

4 2011). Mirroring of tactile components of observed actions indicated in the present

5 study has also previously been suggested by findings showing activation of

6 somatosensory cortices during the observation of hand actions (Avikainen, Forss, \&

7 Hari, 2002; Rossi et al., 2002; Cheyne et al., 2003; Bufalari et al., 2007; Pihko,

8 Nangini, Veikko, \& Hari, 2010). The fact that repetition effects were also observed in

9 the Tactile-Arrows condition of Experiment 1, even when no tactile stimulation was

10 observed, concurs with findings of somatosensory activation during the perception

11 of graspable objects (Proverbio, Adorni, \& D'Aniello, 2011) and is consistent with

12 the findings of stronger mu attenuation during observation of graspable tools than

13 non-tool objects (Proverbio, 2012).

15 A second objective of this study was to investigate if mu attenuation observed

16 during action observation and execution (e.g. Cochin et al., 1999;

17 Muthukumaraswamy \& Johnson 2004a, 2004b; Oberman et al., 2005; Ulloa \&

18 Pineda, 2007; Cheng et al., 2008) is due solely to mirroring of tactile features, or

19 whether it also reflects mirror processing of the motor features as assumed in the

20 previous literature. If mu attenuation reflects motor mirroring, then repetition

21 effects should be sensitive to repetition of the motor, rather than tactile,

22 components of action. Mirroring of tactile features of action would be indicated if

23 repetition effects are observed when tactile, rather than motor, components are the 
1 object of repetition. Experiment 2 showed that repetition effects are limited to the

2 tactile components of actions, by comparing action conditions in which the motor

3 components, tactile components or both were the focus of the repetition. Cross-

4 modal repetition effects were again found in the mu signal, but only in conditions

5 where the tactile components were repeated.

6

7 The finding that these repetition effects appear sensitive to tactile rather than motor

8 components of action suggests that they are due to activity of the human tactile,

9 rather than motor, mirror system. It is commonly assumed that mu attenuation

10 observed over sensorimotor areas during action execution and observation is

11 associated with activity that allows the understanding of observed actions by

12 activation of one's own motor codes (e.g. Cochin et al., 1999; Muthukumaraswamy \&

13 Johnson, 2004a, 2004b; Muthukumaraswamy et al., 2004; Pineda, 2005; Oberman et

14 al., 2005, 2008; Lepage \& Théoret, 2006; Ulloa \& Pineda, 2007; Cheng et al., 2008;

15 Ferrari et al., 2012). However, the present findings provide no evidence to support

16 the hypothesis that mu attenuation during action observation is due to motor

17 mirror processes, and caution should therefore be exercised when making such

18 claims.

20 It should be noted that if repetition effects were observed in the 'Motor-Only'

21 condition of Experiment 2 then they could have been driven by motor processing

22 within premotor or primary motor cortical areas, and / or by somatosensory

23 activity relating to the encoding of proprioceptive information (e.g. Prud'homme \& 
1 Kalaska, 1994). However, no repetition effects were observed in this condition,

2 suggesting that neither motor nor proprioceptive mirroring was reflected in mu

3 attenuation. It could instead be argued that repetition effects were not observed in

4 the Motor-Only condition because mimed actions were used as stimuli, and early

5 papers suggested that single cell responses within premotor cortex to observed

6 actions decrease when observing mimed actions (Gallese, Fadiga, Fogassi, \&

7 Rizzolatti, 1996). However, there is now considerable evidence that mimed actions

8 are mirrored in premotor cortex (e.g. Kraskov, Dancause, Quallo, Shepherd, \&

9 Lemon, 2009; Press, Catmur et al., 2012), and the magnitude of the observed

10 repetition effect was equivalent in the Motor+Tactile and Tactile-Only blocks,

11 providing no evidence for a motor contribution to the mu effects. Nevertheless, one

12 should not use such evidence to draw strong conclusions about the absence of

13 motor mirroring.

15 There are likely attentional consequences of presenting chains of predictable events,

16 given that attention is more likely to be directed towards surprising events (Itti \&

17 Baldi, 2009). However, care was taken to reduce the possibility that these could

18 contribute towards repetition effects in the present study. First, participants with

19 large numbers of exclusions and blocks with execution mistakes were removed from

20 analysis (also note that execution mistakes were equivalent in Repeat and Non-

21 Repeat blocks). Second, unimodal repetition was equated in the two block types.

22 Therefore, although a cue to squeeze a ball for the sixth time may be processed to a

23 lesser extent than a similar cue presented for the first time, this repetition was 
1 equated across our Repeat vs Non-Repeat comparison. Third, within the context of

2 the experiment, Repeat and Non-Repeat events are equally likely. Nevertheless,

3 future systematic examination of effects of stimulus predictability on locus of

4 attention and repetition phenomena (see Segaert et al., 2013) will shed further light

5 on this potentially complex interaction.

6

7 Experiments 1 and 2 observed different effects of repetition; in Experiment 1

8 repetition was associated with a decrease in mu attenuation while repetition led to

9 greater attenuation in Experiment 2. Therefore, the results of Experiment 1 are

10 more in line with classical findings that demonstrate repetition suppression when

11 events activating the same representation are presented in close succession.

12 However, repetition enhancement effects, like those observed in Experiment 2, have

13 also been observed widely in the neuroimaging literature (see Segaert et al., 2013

14 for a discussion). The physiological mechanisms underlying repetition effects are

15 currently a matter of debate, for both fMRI and EEG (Grill-Spector et al., 2006,

16 Oosterhof et al., 2013; Caggiano et al., 2013; Segaert et al., 2013). Further unknown

17 are the factors which determine whether repetition enhancement or repetition

18 suppression is seen, although it is known that the direction of repetition effects is

19 influenced by cognitive factors (such as explicit memory retrieval and attention-

20 related factors), that repetition effects can change from enhancement to suppression

21 and vice versa, and that both suppression and enhancement can be seen in the same

22 area (see Segaert et al. 2013 for a summary). 
1 We speculate that the fact participants needed to select between different action

2 types in Experiment 2, but not Experiment 1, may have generated the different

3 effects (see Press, Weiskopf et al. 2012). Selection between two action types

4 (Experiment 2) - rather than consistent performance of the same action

5 (Experiment 1) - is likely to have resulted in different timing of action relative to

6 stimulus in the two experiments, and timing is known to be crucial in determining

7 the direction of repetition effects (Segaert et al. 2013). For example, action

8 preparation may have been faster in Experiment 1 than 2 given that participants

9 were required to perform the same action rather than select between different

10 actions. Under this account, suppression effects may be seen when executed events

11 follow observed events in close temporal succession, with enhancement seen when

12 there is a greater delay between events (Segaert et al., 2013; note also that in

13 principle the timing implemented may have been more optimal for detecting tactile

14 than motor effects in the current studies). Of course this account is speculative and

15 future research should explicitly manipulate the temporal profile of events in order

16 to test this hypothesis.

18 The relationship between EEG and BOLD repetition effects and those seen at the

19 single neuron level are also presently unclear. Specifically in the case of motor

20 mirroring mechanisms, there has recently been much debate concerning whether

21 the firing rate of mirror neurons does (Kilner et al., 2014), or does not (Caggiano et

22 al., 2013), change with repetition (although note that repetition effects at the level of

23 local field potentials - which generate the EEG signal - are found in both of these 
1 studies). Regardless of these debates, the presence of repetition effects in

2 neuroimaging modalities is thought to evidence selectivity (Perry and Bentin, 2009;

3 Oosterhof et al., 2013; Grill-Spector et al., 2006), and therefore a crossmodal

4 repetition design is appropriate for the purposes of the current experiment.

\section{6. Conclusions}

9 In conclusion, the present study is the first, to our knowledge, to show crossmodal

10 mirror repetition effects in EEG, and evidence for specific tactile mirroring with any

11 neuroimaging measure. Results indicate that attenuation of the central mu rhythm is

12 indeed an index of mirror processes, but suggest the mirror processes they index

13 may be tactile rather than motor. These findings call for caution to be employed

14 when interpreting the results from previous (or future) studies using mu

15 attenuation as an index of motor mirroring. 
1 Conflict of interest: The authors declare no conflict of interest.

3 Acknowledgements: The authors would like to thank Nick Berggen and Max

4 Owens for their technical help, and James Kilner for comments on an earlier version

5 of the manuscript. M.-P. Coll is funded by a doctoral scholarship from the Canadian

6 Institutes of Health Research and received a Michael-Smith supplement for foreign

7 study to accomplish this research. G Bird worked on the manuscript while a Senior

8 Fellow at the Netherlands Institute for Advanced Studies in the Humanities and

9 Social Sciences. C. Catmur is supported by the Economic and Social Research Council

10 (ES/K00140X/1). 
2 References

3 Arnstein, D., Cui, F., Keysers, C., Maurits, N. M., \& Gazzola, V. (2011). $\mu$ -

4 suppression during action observation and execution correlated with BOLD in

5 dorsal premotor, inferior parietal and SI cortices. The Journal of Neuroscience,

6 31(40), 14243-14249.

7 Avikainen, S., Forss, N., \& Hari, R. (2002). Modulated activation of the human

8 SI and SII cortices during observation of hand actions. Neuroimage, 15(3), 640-646.

9 Babiloni, C., Del Percio, C., Rossini, P. M., Marzano, N., Iacoboni, M., Infarinato,

10 F., ... Eusebi, F. (2009). Judgment of actions in experts: a high-resolution EEG study

11 in elite athletes. Neuroimage, 45(2), 512-521.

12 Banissy, M. J. \& Ward, J. (2007). Mirror-touch synesthesia is linked with

13 empathy. Nature Neuroscience, 10, 815-816.

14 Blakemore, S. J., Bristow, D., Bird, G., Frith, C., \& Ward, J. (2005).

15 Somatosensory activations during the observation of touch and a case of vision-

16 touch synaesthesia. Brain, 128(7), 1571-1583.

17 Bufalari, I., Aprile, T., Avenanti, A., Di Russo, F., \& Aglioti, S.M. (2007).

18 Empathy for pain and touch in the human somatosensory cortex. Cerebral Cortex,

19 17(11), 2553-2561.

20 Caggiano, V., Pomper, J. K., Fleischer, F., Fogassi, L., Giese, M., \& Their, P.

21 (2013). Mirror neurons in monkey area F5 do not adapt to the observation of

22 repeated actions. Nature Communications, 4, 1433 
Cheng, Y., Lee, P. L., Yang, C. Y., Lin, C. P., Hung, D., \& Decety, J. (2008). Gender

2 differences in the mu rhythm of the human mirror-neuron system. PLoS One, 3,

3 e2113.

4 Cheyne, D., Gaetz, W., Garnero, L., Lachaux, J. P., Ducorps A., Schwartz, D., \&

5 Varela, F. J. (2003). Neuromagnetic imaging of cortical oscillations accompanying

6 tactile stimulation. Cognitive Brain Research, 17(3), 599-611.

7 Chong, T. T., Cunnington, R., Williams, M. A., Kanwisher, N., \& Mattingley, J. B.

8 (2008). fMRI adaptation reveals mirror neurons in human inferior parietal cortex.

$9 \quad$ Current Biology, 18(20), $1576-1580$.

10 Cochin, S., Barthelemy, C., Roux, S., \& Martineau, J. (1999). Observation and

11 execution of movement: similarities demonstrated by quantified

12 electroencephalography. European Journal of Neuroscience, 11(5), 1839-1842.

13 Cook, R., Bird, G., Catmur, C., Press, C., \& Heyes, C. (2014). Mirror neurons:

14 From origin to function. Behavioral and Brain Sciences, 37(2), 177-192.

15 Ebisch, S. J., Perruci, M. G., Ferretti, A., Del Gratta, C., Romani, G. L., \& Gallese,

16 V. (2008). The sense of touch: embodied simulation in a visuotactile mirroring

17 mechanism for observed animate or inanimate touch. Journal of Cognitive

18 Neuroscience, 20(9), 1611-1623.

19 Ferrari, P. F., Vanderwert, R. E., Paukner, A., Bower, S., Suomi, S. J., \& Fox, N. A.

20 (2012). Distinct EEG amplitude suppression to facial gestures as evidence for a

21 mirror mechanism in newborn monkeys. Journal of Cognitive Neuroscience, 24(5),

$22 \quad 1165-1172$. 
Gaetz, W. \& Cheyne, D. (2006). Localization of sensorimotor cortical rhythms

2 induced by tactile stimulation using spatially filtered MEG. Neuroimage, 30(3), 899-

3908.

4 Gallese, V., Fadiga, L., Fogassi, L., \& Rizzolatti, G. (1996). Action recognition in

5 the premotor cortex. Brain, 119(2), 593-609.

6 Garrido, M. I., Kilner, J. M., Kiebel, S. J., Stephan, K. E., Baldeweg, T., \& Friston,

7 K. J. (2009). Repetition suppression and plasticity in the human brain. Neuroimage, 8 48(1), 269-279.

9 Grill-Spector, K., Henson, R., \& Martin, A. (2006). Repetition and the brain:

10 neural models of stimulus-specific effects. Trends in Cognitive Sciences, 10(1), 14-23.

11 Hari, R. (2006). Action-perception connection and the cortical mu rhythm.

12 Prog Brain Res, 159, 253-260.

13 Hari, R., Forss, N., Avikainen, S., Kirveskari, E., Salenius, S., \& Rizzolatti, G.

14 (1998). Activation of human primary motor cortex during action observation: a 15 neuromagnetic study. Proc Natl Acad Sci USA, 95(25), 15061-15065.

16 Hari, R., \& Salmelin, R. (1997). Human cortical oscillations: a neuromagnetic

17 view through the skull. Trends in neurosciences, 20(1), 44-49.

18 Höfle, M., Pomper, U., Hauck, M., Engel, A. K., \& Senkowski, D. (2013). Spectral

19 signatures of viewing a needle approaching one's body when anticipating pain.

20 European Journal of Neuroscience, 38(7), 3089-3098.

21 Itti, L., \& Baldi, P. (2009). Bayesian surprise attracts human attention. Vision

22 Research, 49(10), 1295-1306. 
Jaspers, H. H. (1958). The ten-twenty electrode system of the International

2 Federation. Electroencephalography and Clinical Neurophysiology, 10, 371-375.

3 Keysers, C., Kaas, J. A., \& Gazzola, V. (2010). Somaotsensation in social

4 perception. Nature Review Neuroscience, 11(6), 417-428.

$5 \quad$ Keysers, C., Wicker, B., Gazzola, V., Anton, J.-L., Fogassi, L., \& Gallese, V.

6 (2004). A touching sight: SII/PV activation during the observation and experience

7 of touch. Neuron, $42(2), 335-346$.

8 Kilner, J., Kraskov, A., \& Lemon, R. (2014). Do monkey F5 mirror neurons

9 show changes in firing rate during repeated observation of natural actions? Journal

10 of Neurophysiology, 111(6), 1214-1226.

11 Kilner, J. M., Neal, A., Weiskopf, N., Friston, K. J., \& Frith C. D. (2009). Evidence

12 of mirror neurons in human inferior frontal gyrus. The Journal of Neuroscience,

$1329(32), 10153-10159$.

14 Klimesch, W. (1999). EEG alpha and theta oscillations reflect cognitive and

15 memory performance: a review and analysis. Brain Research Reviews, 29(2-3), 169-

16195.

17 Kraskov, A., Dancause, N., Quallo, M. M., Shepherd, S., \& Lemon, R. N. (2009).

18 Corticospinal neurons in macaque ventral premotor cortex with mirror properties: a

19 potential mechanism for action suppression? Neuron, 64(6), 922-930.

20 Kuehn, E., Muller, K., Turner, R., \& Schütz-Bosbach, S. (2014). The functional

21 architecture of S1 during touch observation described with 7T fMRI. Brain Structure

22 and Function, 219(1), 119-140. 
Lepage, J. F. \& Théoret, H. (2006). EEG evidence for the presence of an action

2 observation-execution matching system in children. European Journal of

3 Neuroscience, 23(9), 2505-2510.

4 Lingnau, A., Gesierich, B., \& Caramazza, A. (2009). Asymmetric fMRI

5 adaptation reveals no evidence for mirror neurons in humans. Proceedings of the

6 National Academy of Sciences USA, 106(24), 9925-9930.

7 Martínez-Jauand, M., González-Roldán, A. M., Muñoz, M. A., Sitges, C., Cifre, I.,

8 \& Montoya, P. (2012). Somatosensory activity modulation during observation of

9 other's pain and touch. Brain Research, 1467, 48-55.

10 Muthukumaraswamy, S. D. \& Johnson, B.W. (2004a). Primary motor cortex

11 activation during action observation revealed by wavelet analysis of the EEG.

12 Clinical Neurophysiology, 115(8), 1760-1766.

13 Muthukumaraswamy, S. D. \& Johnson, B. W. (2004b). Changes in rolandic mu

14 rhythm during observation of a precision grip. Psychophysiology, 41(1), 152-156.

15 Muthukumaraswamy, S. D., Johnson, B. W., \& McNair, N. A. (2004). Mu

16 rhythm modulation during observation of an object-directed grasp. Cognitive Brain

17 Research, 19(2), 195-201.

18 Oberman, L. M., Hubbard, E. M., McCleery, J. P., Altschuler, E. L.,

19 Ramachandran, V. S., \& Pineda, J. A. (2005). EEG evidence for mirror neuron

20 dysfunction in autism spectrum disorders. Cognitive Brain Research, 24(2), 190-198.

21 Oberman, L. M., Pineda, J. A., \& Ramachandran, V. S. (2006). The human

22 mirror neuron system: A link between action observation and social skills. Social

23 Cognitive and Affective Neuroscience, 2(1), 62-66. 
Oberman, L. M., Ramachandran, V. S., \& Pineda, J. A. (2008). Modulation of mu

2 suppression in children with autism spectrum disorders in response to familiar or

3 unfamiliar stimuli: The mirror neuron hypothesis. Neuropsychologia, 46(5), 1558-

41565.

5 Oldfield, R. C. (1971). The assessment and analysis of handedness: The

6 Edinburgh inventory. Neuropsychologia, 9, 81-113.

7 Oosterhof, N. N., Tipper, S. P., \& Downing, P. E. (2013). Crossmodal and

8 action-specific: neuroimaging the human mirror neuron system. Trends in Cognitive

$9 \quad$ Sciences, 17(7), 311- 318.

10 Ortigue, S., Thompson, J.C., Prasuraman, R., \& Grafton, S. (2009). Spatio-

11 temporal dynamics of human intention understanding in temporo-parietal cortex: A

12 combined EEG/fMRI repetition suppression paradigm. PLoS One, 4(9), e6962.

13 Perry, A., \& Bentin, S. (2009). Mirror activity in the human brain while

14 observing hand movements: A comparison between EEG desynchronization in the

15 mu range and previous fMRI results. Brain Research, 1282(28), 126-132.

16 Perry, A., Bentin, S., Bartal, I. B. A., Lamm, C., \& Decety, J. (2010). “Feeling” the

17 pain of those who are different from us: Modulation of EEG in the mu/alpha range.

18 Cognitive, Affective and Behavioral Neuroscience, 10(10), 493-504.

19 Pihko, E., Nangini, C., Veikko, J., \& Hari, R. (2010). Observing touch activates

20 human primary sensory cortex. European Journal of Neuroscience, 31(10), 1836-

2121843.

22 Pineda, J. A. (2005). The functional significance of mu rhythms: translating

23 "seeing" and "hearing" into "doing". Brain Research Reviews, 50(1), 57-68. 
Press, C., Weiskopf, N., \& Kilner, J. M. (2012). Dissociable roles of human

2 inferior frontal gyrus during action execution and observation. Neuroimage, 60(3),

$3 \quad 1671-1677$.

4 Press, C., Catmur, C., Cook, R., Widmann, H., Heyes, C., \& Bird, G. (2012). FMRI

5 evidence of 'mirror' responses to geometric shapes. PLoS One, 7, e51934.

6 Proverbio, A. M., Adorni, R., \& D’Aniello, G. E. (2011). 250 ms to code for

7 action affordance during observation of manipulable objects. Neuropsychologia,

$8 \quad 49(9), 2711-2717$.

9 Proverbio, A. M. (2012). Tool perception suppresses 10-12 Hz mu rhythm of

10 EEG over the somatosensory area. Biological Psychology, 91(1), 1-7.

11 Prud'homme, M. J. \& Kalaska, J. F. (1994). Proprioceptive activity in primate

12 primary somatosensory cortex during active arm reaching movements. Journal of

13 Neurophysiology, 72(5), 2280-2301.

14 Ritter, P., Moosmann, M., \& Villringer, A. (2009). Rolandic alpha and beta EEG

15 rhythms' strengths are inversely related to fMRI-BOLD signal in primary

16 somatosensory and motor cortex. Human Brain Mapping, 30(4), 1168-1187.

17 Rizzolati, G., Fabbri-Destro, M., \& Cattaneo, L. (2009). Mirror neurons and

18 their clinical relevance. Nature Reviews Neurology, 5, 24-34.

19 Rossi, S., Tecchio, F., Pasqualetti, P., Ulivelli, M., Pizzella, V., Romani, G. L., ...

20 Rossini, P. M. (2002). Somatosensory processing during movement observation in

21 humans. Clinical Neurophysiology, 113(1), 16-24. 
2 events: Interindividual differences and vicarious somatosensory responses during

3 touch observation. NeuroImage, 60(2), 952-957.

4 Segaert, K., Weber, K., de Lange, F. P., Petersson, K. M., \& Hagoort, P. (2013).

5 The suppression of repetition enhancement: a review of fMRI studies.

6 Neuropsychologia, 51(1), 59-66.

7 Ulloa, E. R. \& Pineda, J. A. (2007). Recognition of point-light biological motion:

8 mu rhythms and mirror neuron activity. Behavioural and Brain Research, 183(2),

$9 \quad 188-194$.

10 van Wijk, B., Willemse, R., \& Vandertop, W. P. (2012). Slowing of M1

11 oscillations in brain tumor patients in resting state and during movement. Clinical

12 Neurophysiology, 123(11), 2212-2219.

13 


\section{Footnote 1}

It is worth noting that the average band is $1 \mathrm{~Hz}$ lower in Experiment 1 than Experiment 2, despite equivalent selected ranges. Frequency bands in both experiments are unambiguously alpha rather than beta range, given selection criteria, and therefore akin to ranges associated with somatosensory rather than motor cortex activation. Such small discrepancies between experiments most likely reflect individual differences, especially given that this difference was not significant $[\mathrm{t}(30)=1.37, \mathrm{p}=0.18, \mathrm{~d}=0.49]$, but may in principle result from changes to the task. 


\section{Table and figure captions}

Table 1. Description of the different mini-block types according to the two experimental factors Condition and Repetition for Experiments 1 and 2.

Table 2. Results from the ANOVAs for both experiments.

Figure 1. Schematic representation of Repeat and Non-Repeat pairs of stimuli from all conditions of Experiments 1 and 2. The stimuli were presented for $1500 \mathrm{~ms}$ and participants always alternated between observation and execution. Ten repeated or non-repeated stimuli were presented in each experimental block, with repetition defined cross-modally (within-modality repetition was equated in Repeat and NonRepeat blocks). Greyed out pictures in the execution trials are present for illustrative purposes only. Asterisks in Experiment 1 indicate the ball covered with a black mesh, for representational purposes only.

Figure 2. (A) Time course of the attenuation relative to baseline as a function of time, Condition and Repetition, averaged over participant-specific mu bands for Experiment 1. (B) Average difference in power between Non-Repeat and Repeat conditions for the 7-14 Hz spectrum, across time, as a function of Condition for Experiment 1. Values above zero indicate stronger attenuation in the Repeat condition. Data were smoothed across time and frequency for display purposes using a $30 \mathrm{~ms}$ and a $3 \mathrm{~Hz}$ wide moving average. 
Figure 3. (A) Time course of the attenuation relative to baseline as a function of time, Condition and Repetition, averaged over participant-specific mu bands for Experiment 2. (B) Average difference in power between Non-Repeat and Repeat conditions for the 7-14 Hz spectrum, across time, as a function of Condition for Experiment 2. Values above zero indicate stronger attenuation in the Repeat condition. Data were smoothed across time and frequency for display purposes using a $30 \mathrm{~ms}$ and a $3 \mathrm{~Hz}$ wide moving average. 


\section{Highlights}

- Crossmodal repetition design to assess tactile and motor mirroring with EEG

- Mu repetition effects support the existence of a tactile mirroring system

- No evidence found that motor mirroring is reflected in the mu signal

- Therefore mu attenuation during action observation may reflect tactile mirroring

- Results question commonly assumed nature of mu effects during action observation 
Table 1

\begin{tabular}{|c|c|c|c|c|}
\hline Experiment & Condition & Repetition & Action observed & Action executed \\
\hline \multirow[t]{8}{*}{ Experiment 1} & \multirow[t]{4}{*}{ Tactile-Action } & \multirow[t]{2}{*}{ Repeat } & Smooth ball squeeze & Smooth ball squeeze \\
\hline & & & Mesh ball squeeze & Mesh ball squeeze \\
\hline & & \multirow[t]{2}{*}{ Non-Repeat } & Smooth ball squeeze & Mesh ball squeeze \\
\hline & & & Mesh ball squeeze & Smooth ball squeeze \\
\hline & \multirow[t]{4}{*}{ Tactile-Arrows } & \multirow[t]{2}{*}{ Repeat } & Arrows towards smooth ball & Smooth ball squeeze \\
\hline & & & Arrows towards mesh ball & Mesh ball squeeze \\
\hline & & \multirow[t]{2}{*}{ Non-Repeat } & Arrows towards smooth ball & Mesh ball squeeze \\
\hline & & & Arrows towards mesh ball & Smooth ball squeeze \\
\hline \multirow[t]{12}{*}{ Experiment 2} & \multirow[t]{4}{*}{ Motor+Tactile } & \multirow[t]{2}{*}{ Repeat } & Ball squeeze & Ball squeeze \\
\hline & & & Pen stroke & Pen stroke \\
\hline & & \multirow[t]{2}{*}{ Non-Repeat } & Ball squeeze & Pen stroke \\
\hline & & & Pen stroke & Ball squeeze \\
\hline & \multirow[t]{4}{*}{ Motor-Only } & \multirow[t]{2}{*}{ Repeat } & Mimed ball squeeze & Mimed ball squeeze \\
\hline & & & Mimed pen stroke & Mimed pen stroke \\
\hline & & \multirow[t]{2}{*}{ Non-Repeat } & Mimed ball squeeze & Mimed pen stroke \\
\hline & & & Mimed pen stroke & Mimed ball squeeze \\
\hline & \multirow[t]{4}{*}{ Tactile-Only } & \multirow[t]{2}{*}{ Repeat } & Ball moving into hand & Ball squeeze \\
\hline & & & Pen moving into hand & Pen stroke \\
\hline & & \multirow[t]{2}{*}{ Non-Repeat } & Ball moving into hand & Pen stroke \\
\hline & & & Pen moving into hand & Ball squeeze \\
\hline
\end{tabular}


Table 2

$\begin{array}{llll}\text { DF } & \text { F } & p & \text { eta }^{2} \text { partial }\end{array}$

\section{Experiment 1}

\begin{tabular}{lcccc} 
Hemisphere & 1,16 & $<1$ & 0.96 & - \\
Condition & 1,16 & 9.04 & $<\mathbf{0 . 0 5}$ & 0.36 \\
Repetition & 1,16 & 5.47 & $<\mathbf{0 . 0 5}$ & 0.26 \\
$\begin{array}{l}\text { Experiment 2 } \\
\text { Hemisphere }\end{array}$ & 1,14 & 3.66 & 0.08 & 0.21 \\
Condition & 2,28 & $<1$ & 0.99 & - \\
Repetition & 1,14 & 3.18 & 0.10 & 0.19 \\
Condition*Repetition & 2,28 & 3.50 & $<\mathbf{0 . 0 5}$ & 0.20 \\
\hline
\end{tabular}


Figure 1

\section{Experiment 1}

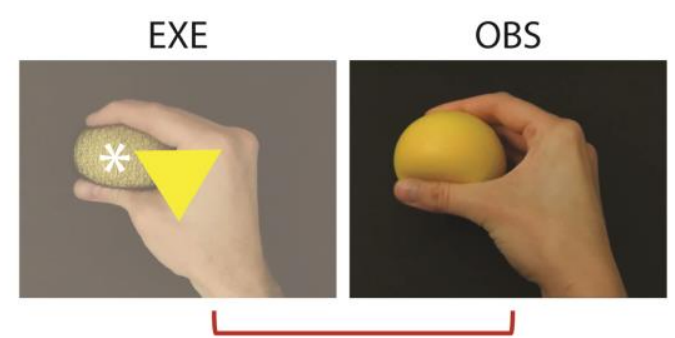

Tactile-Action Non-Repeat
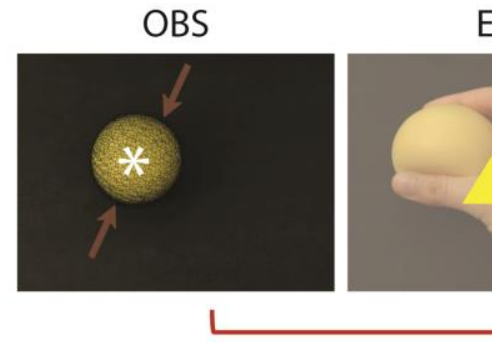

Tactile-Arrows Non-Repeat

\section{Experiment 2}

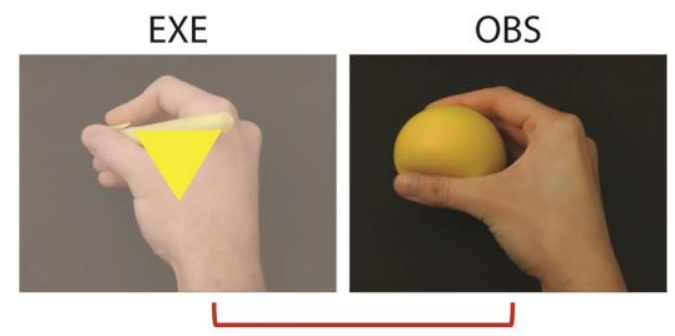

Motor+Tactile Non-Repeat
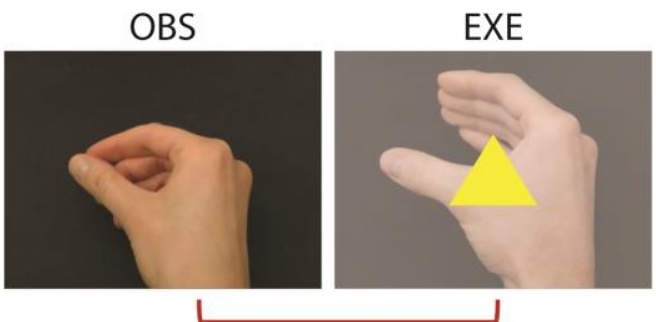

Motor-Only Non-Repeat
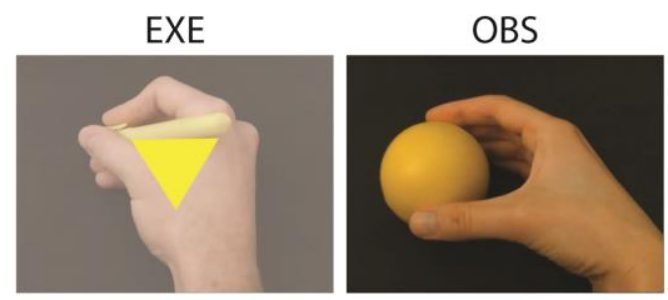

Tactile-Only Non-Repeat

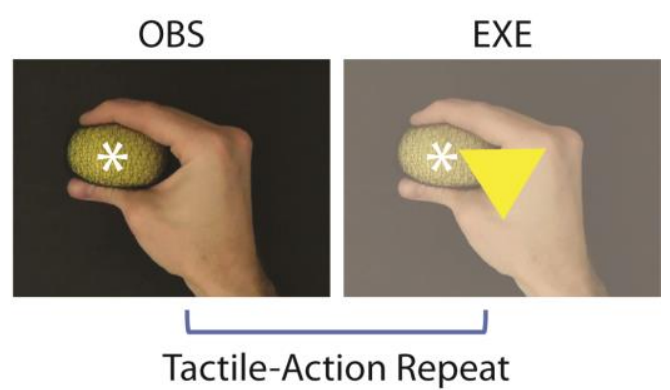

EXE
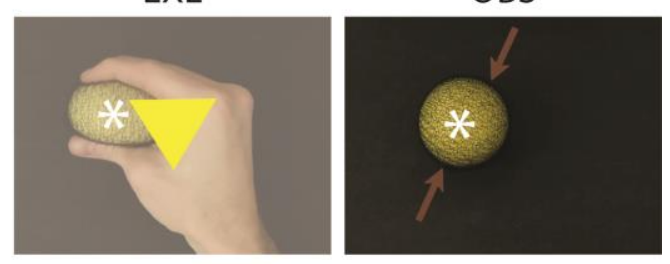

Tactile-Arrows Repeat

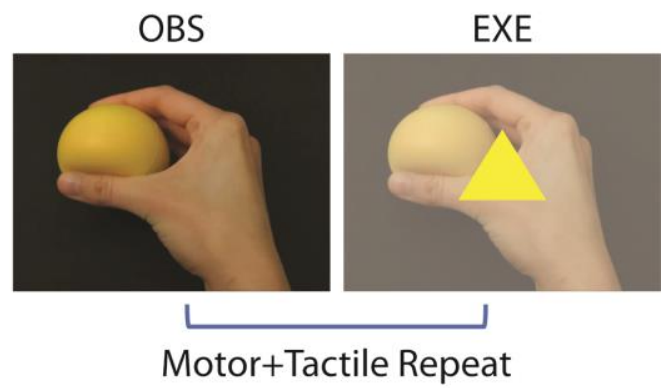

EXE

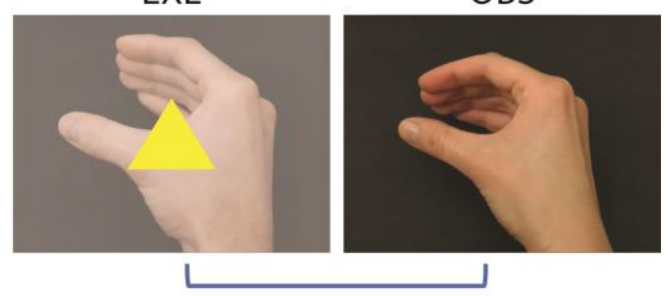

Motor-Only Repeat
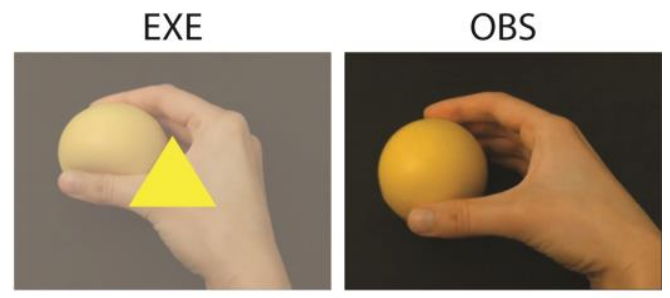

Tactile-Only Repeat 


\section{Figure 2}

A
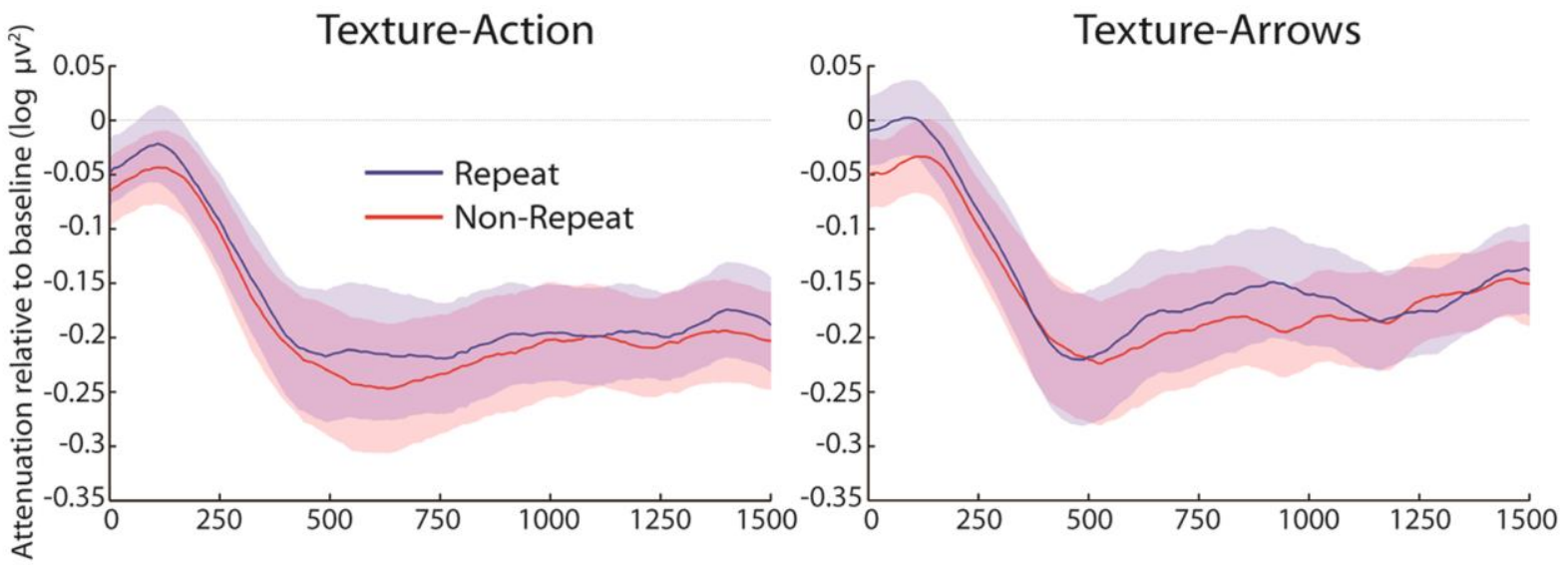

B
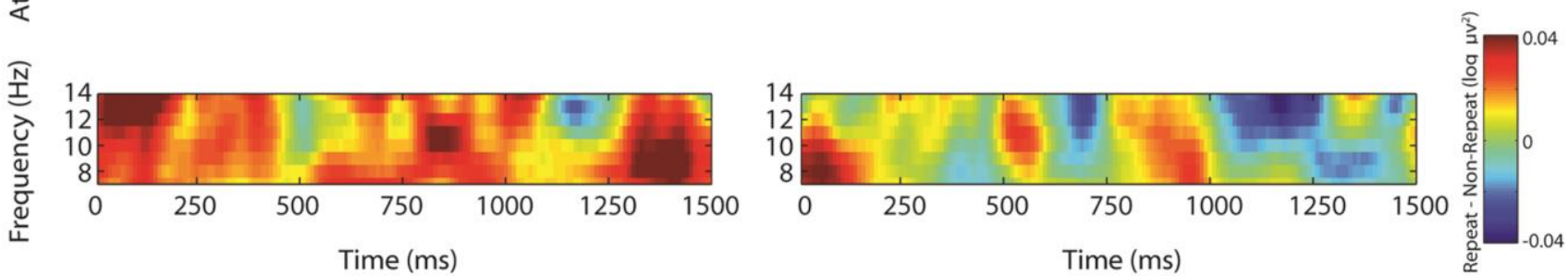
Figure 3

A
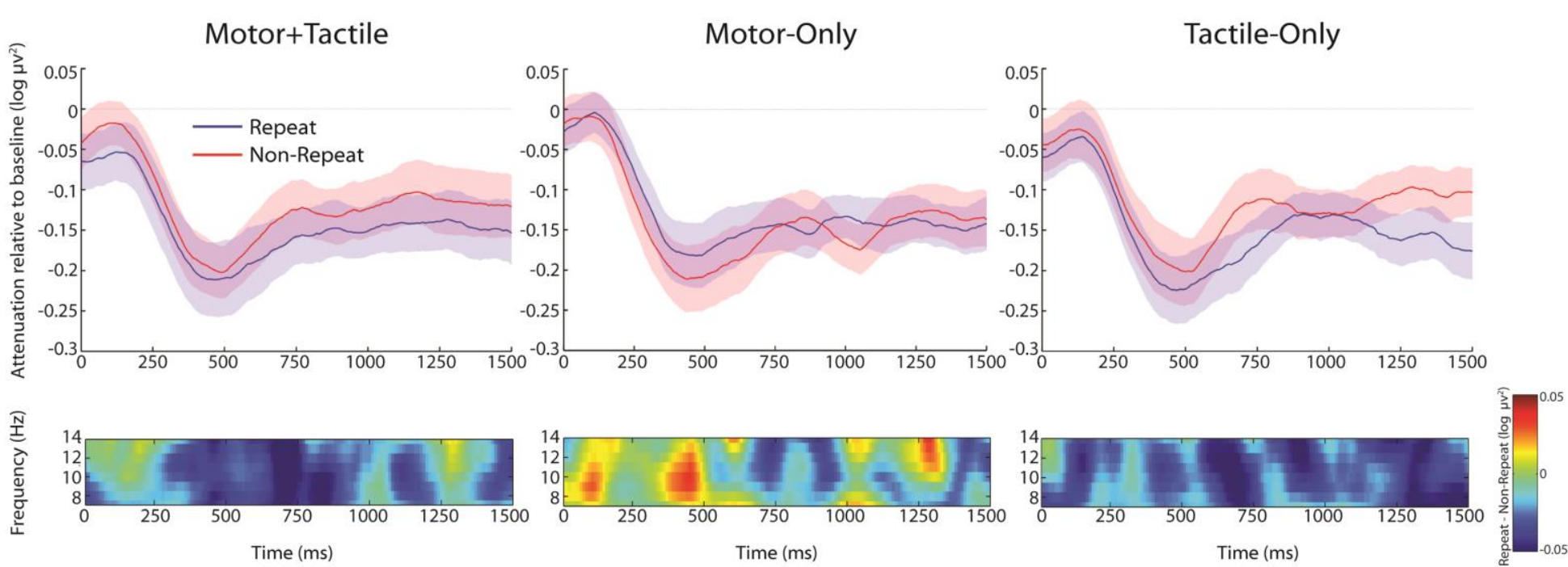